\title{
Redes Cognitivas Dinâmicas, Fundamentos e Aplicações
}

\section{Dynamic Cognitive Networks, Fundamentals and Applications}

\author{
Márcio Mendonça ${ }^{1}$; Lúcia V. Ramos de Arruda ${ }^{2}$; \\ Rodrigo Henrique C. Palácios ${ }^{3}$; Wagner F. Godoy ${ }^{4}$
}

\begin{abstract}
Resumo
Este trabalho apresenta uma proposta de redes cognitivas dinâmicas, do inglês Dynamic Cognitive Networks (DCN), evolução dos Mapas Cognitivos e dos Mapas Cognitivos Fuzzy. Os Mapas Cognitivos Fuzzy ou Fuzzy Cognitive Maps (FCM) têm aplicações em diversas áreas de conhecimento, entretanto apresentam algumas restrições para sistemas dinâmicos. Devido a essas restrições existem na literatura algumas propostas de arquiteturas baseadas nos FCM clássicos e propostas de modelos cognitivos baseados nessa ferramenta, Redes Cognitivas Dinâmicas é uma dessas abordagens. Este trabalho apresenta uma proposta original com fundamentos para a construção de uma DCN e aplicações em controle de processos e navegação autônoma.
\end{abstract}

Palavras-chave: Redes cognitivas dinâmicas. Mapas cognitivos Fuzzy. Navegação autônoma.

\begin{abstract}
This study presents the proposal of dynamic cognitive networks (DCN), and also the evolution of Cognitive Maps and Fuzzy Cognitive Maps. Fuzzy Cognitive Maps (FCM) can be applied in several areas of knowledge; however, it presents some restrictions in dynamic systems. Due to these restrictions, some architectures proposals are based on FCM and also classical proposals for cognitive models based on these concepts are available in the literature. Dynamic Cognitive Networks is one of these approaches. Hence, this study presents an original proposal with background for the construction of DCN and applications in process control and autonomous navigation.
\end{abstract}

Keywords: Dynamic Cognitive Networks, Fuzzy Cognitive Maps, Autonomous Navigation.

1 Departamento de Engenharia Elétrica, Universidade Tecnológica Federal do Paraná, Cornélio Procópio, Paraná, Brasil, mendonca@utfpr.edu.br.

2 Laboratório de Automação e Sistemas de Controle Avançado, Universidade Tecnológica Federal do Paraná, Curitiba, Paraná, Brasil, lvrarruda@utfpr.edu.br.

3 Departamento de Engenharia de Computação, Universidade Tecnológica Federal do Paraná, Cornélio Procópio, Paraná, Brasil, rodrigopalacios@utfpr.edu.br.

4 Departamento de Engenharia Elétrica, Universidade Tecnológica Federal do Paraná, Cornélio Procópio, Paraná, Brasil, wagnergodoy@utfpr.edu.br. 


\section{Introdução}

A Inteligência Artificial (AI) envolvem métodos que tentam imitar as capacidades dos seres humanos para resolver problemas, devido à possibilidade de lidar com processos complexos, baseados em informações imprecisas e/ou aproximadas, que podem ser adquiridas a partir dos dados de operação e/ou funcionamento de um sistema. Entre as técnicas inteligentes, as redes neurais se destacam pela capacidade de processar dados massivos de uma maneira paralela (HAYKIN, 2000), ou pela aquisição de conhecimento de um ou mais especialistas por meio de sistemas Fuzzy (KOSKO, 1986). Por outro lado, o mapa cognitivo Fuzzy, do inglês Fuzzy Cognitive Map (FCM), é uma ferramenta para a modelagem do conhecimento humano, obtido através de termos linguísticos, inerentes aos sistemas Fuzzy, mas com uma estrutura semelhante às Redes Neurais Artificiais (RNA), a qual facilita o tratamento de dados e possui capacidade de treinamento e adaptação. De modo resumido, mapas cognitivos Fuzzy é uma técnica baseada no conhecimento que funciona como uma rede cognitiva artificial e herda características de mapas cognitivos e redes neurais artificiais (GLYKAS, 2010). O FCM pode ser considerado um modelo híbrido (fusão de duas áreas de conhecimento de sistemas inteligentes) com um forte grau de iteração, no qual não se pode identificar e separar as estruturas das áreas de origens, somente a semântica de construção (GHAZANFARI et al., 2007).

Além das vantagens e características herdadas das técnicas primárias, FCM foi originalmente proposto como uma ferramenta para construção de modelos e/ou mapas cognitivos em diversas áreas de conhecimento, o que sugere que a técnica confere uma facilidade na abstração de informações necessárias à modelagem de sistemas complexos, devido à sua semelhança de construção com o raciocínio humano. Deste modo, os mapas cognitivos Fuzzy agregam vantagens de aquisição, tratamento e capacidade de adaptação a partir de dados e informações do sistema a ser modelado, com uma capacidade inteligente de tomada de decisão devido à sua natureza heurística. Entretanto, os FCM apresentam dificuldade de modelagem de tempo, restringindo para aplicações onde as causas e efeitos ocorrem de modo simultâneo. Assim, esse artigo apresenta uma proposta de evolução do FCM, que agrega a ocorrência de eventos e os torna apropriados, a priori, para desenvolvimento de sistemas inteligentes de controle e automação.

\section{Revisão DCN e FCM}

Desde o trabalho pioneiro de Kosko (1986), que estendeu os mapas cognitivos de Axerold (1976) para a inclusão da lógica Fuzzy, várias aplicações de FCM são reportadas na literatura em diversas áreas do conhecimento. Em especial, citam-se as aplicações em vida artificial (DICKERSON; KOSKO, 1994), tomada de decisão em rodovias de acesso rápido (PERUSICH, 1996), modelagem e tomada de decisão em ambientes corporativos e comércio eletrônico (LEE; LEE, 2003), tomada decisão na área médica (PAPAGEORGIOU; STYLIOS; GROUMPOS, 2007).

Os FCM são capazes de representar o conhecimento e implementar os operadores de inferência com maior flexibilidade, em comparação com muitos outros métodos disponíveis. No entanto, apesar desses benefícios, FCM convencionais não são capazes de lidar com o conceito de tempo de forma direta. Em consequência disso, sistemas políticos, mecânica, eletrônica, entre outros têm inevitavelmente comportamentos dinâmicos que os FCM são incapazes de modelar. (ACAMPORA; LOIA, 2011). Neste contexto, diversas propostas baseadas no FCM clássico são encontradas na literatura.

De acordo com o paragrafo anterior, diversas abordagens conhecidas na literatura utilizam modelos combinados entre FCM e outras ferramentas computacionais inteligentes, como por exemplo, DT-FCM (Decision Tree - FCM) 
um modelo cognitivo híbrido que utiliza árvores de decisão e FCM (PAPAGEORGIOU; STYLIOS; GROUMPOS, 2006). Observa-se que estes trabalhos também apresentam variações semânticas da proposta original de FCM, adaptando a estrutura do FCM de acordo com o problema tratado. De modo mais específico, os trabalhos sequentes são algumas das propostas conhecidas na literatura como evolução dos mapas cognitivos, assim como os FCM, em especial as propostas de evolução do FCM, foco deste trabalho.

Outra ferramenta baseada em mapas cognitivos encontrada na literatura é a Fuzzy-SDG (Signed Direct Graph). Esta topologia utiliza o conhecimento de especialistas na forma estruturada e é abstraído através do desenvolvimento de um mapa cognitivo. Os conceitos dão origem a variáveis Fuzzy e as relações causais originam uma base de regras do tipo antecedente-consequente. O trabalho de Ghazanfari, Alizadeh e Jafari (2002) desenvolve um sistema dinâmico de compras para exemplificar a proposta. Esse sistema inteligente modela o tempo através do disparo de regras e inclui um fator de probabilidade para cada regra modelada. A probabilidade objetiva atenuar fatores como: incertezas, imprecisões e ambiguidade, inerentes ao processo de aquisição de conhecimento indutivo.

O trabalho de Huang e Wang (1999) apresenta uma FCN (Fuzzy Causal Network) como uma proposta de evolução do Fuzzy-SDG. Essa ferramenta também emprega o conhecimento dos especialistas para o desenvolvimento de um mapa cognitivo, ou seja, atribuem conceitos como variáveis do problema e modelam as relações causais como correlação entre variáveis. Contudo, os autores citam as dificuldades na identificação e quantificação das relações causais. Como solução, os autores propõem uma divisão da FCN em sub-redes menores e aplicam o treinamento supervisionado backpropagation para obtenção da magnitude das relações causais modeladas. Para exemplificar a ferramenta, os autores empregam a FCN em controle de processos, num sistema de tratamento de águas residuais.
Em Zhang, Liu e Zhou (2006), é apresentado outro tipo de FCN em que o mecanismo de inferências se dá através da criação e ativação dinâmica de subconjuntos dos conceitos modelados. Esses conjuntos e subconjuntos são contidos em intervalos mínimos e máximos das variáveis modeladas (conceitos) e, de acordo com regras de funcionamento, são ativados considerando também sua evolução temporal. Os autores consideram a ferramenta desenvolvida ideal para representação de sistemas dinâmicos. Os autores utilizam, como exemplo de aplicação da ferramenta, um sistema de tomada de decisões para gerenciamento de uma universidade baseada em políticas internas e conhecimento de especialistas de diferentes departamentos.

Como uma combinação de FCM e FCN surgem em seguida as DCN (redes cognitivas dinâmicas) que pretende combinar as propriedades do FCM aos sistemas de inferência causal das FCN. A DCN descrita em Miao et al. (2001) é uma das primeiras propostas de DCN e utilizam funções de transferência no domínio de Laplace para representar o comportamento dinâmico das relações causais de um mapa cognitivo. Cada conceito de uma DCN tem seu valor calculado a partir de uma função de transferência ou de um modelo em espaço de estado. Estruturalmente uma DCN é mais flexível que um FCM. De um modo geral, uma DCN pode ser tão simples quanto um mapa cognitivo (CM), um FCM, ou tão complexo como um sistema dinâmico não-linear. Para demonstrar o potencial da ferramenta, um sistema de estratégia e tomada de decisão financeira para gerenciamento de plantações é construído e resultados simulados são apresentados e comparados com um FCM.

O trabalho de Koulouriotis et al. (2005) apresenta uma proposta de DCN para aproximação de séries temporais aplicada em sistemas financeiros. Segundo os autores os FCM têm limitações para modelagem de sistemas complexos principalmente devido a não-linearidades e fenômenos temporais. A arquitetura proposta desse trabalho é semelhante 
à proposta de Miao et al. (2001), na medida em que é obtida a partir de FCM. Entretanto, a DCN proposta tem mais semelhança com RNA uma vez que usa o algoritmo de Levenberg-Marquardt para treinamento e/ou sintonia da rede cognitiva. Os autores validam e concluem a proposta através de comparativos entre DCN e RNA.

Uma nova proposta de mapa cognitivo dinâmico é apresentada em Kottas, Boutallis e Christodoulou (2007) e denominada rede cognitiva Fuzzy (Fuzzy Cognitive Network - FCN). Essas FCN são semelhantes às DCN e utilizam um mecanismo de atualização da matriz $\mathrm{W}$ baseado no algoritmo do gradiente (regra delta) a partir da realimentação do erro medido entre o estado atual e o estado desejado da rede. Os autores aplicam a FCN projetada no controle de nível de um modelo simplificado de hidroelétrica. Como a DCN proposta por Koulouriotis et al. (2005), essas FCN estão mais próximas de uma RNA do que de um mapa cognitivo.

A fim de estabelecer um estado da arte, Miao et al. (2010) apresentaram a transformação de CM em FCM e de FCM para DCN. Esses autores utilizam uma DCN simplificada (Simplified Dynamic Cognitive Network - sDCN) aplicada ao problema de ameaça terrorista, o qual foi utilizado originalmente por Kosko (1986) para exemplificar a evolução de CM, FCM até a sDCN. A sDCN usa conceitos e relações causais fuzzificadas (com a aquisição de conhecimento semelhante ao FCM) para cálculo dos valores numéricos das relações e conceitos. Entretanto, a sDCN é capaz de modelar conhecimento não monotônico, ou seja, tomar conclusões diferentes conforme ocorrem mudanças no sistema modelado. O exemplo apresentado a seguir, de tomada de decisão, realizada pela sDCN exemplifica essa característica. No modelo de ameaça terrorista é possível viajar ou adiar a viagem de acordo com mudanças no nível de ameaça terrorista e/ou a necessidade de realização de negócios. Para executar esse exemplo de inferência é criado um conjunto de valores para as relações e os conceitos inicialmente modelados. A transição entre os estados dos valores das relações e dos conceitos são realizados por funções de tomadas de decisões, conforme necessidade do sistema modelado. Em seguida os autores mostram a DCN com relações causais expressas por meio de funções de transferência de Laplace, como uma evolução das SDCN.

Do exposto anteriormente, existem diferentes arquiteturas que combinam algoritmos tais que RNA, regras de decisão Fuzzy, algoritmos evolutivos e populacionais, funções de probabilidade entre outros ao FCM na busca de um sistema autônomo e inteligente principalmente para tomada de decisão. No entanto, não existe ainda uma solução fechada e definitiva sobre uma estrutura eficiente, robusta e flexível para esse tipo de sistema. Num primeiro momento, propõe-se a utilização de metaheurísticas na otimização das relações causais dos FCM e, em especial, o uso de técnicas de aprendizagem por reforço como uma proposta de adaptação dinâmica, semelhante às apresentadas em Koulouriotis, Diakoulakis e Emiris (2001). Neste contexto, uma proposta de uma arquitetura cognitiva de forma estruturada é então expandida pela inclusão de novos tipos de conceitos e relações, e não apenas aquelas do tipo causa-efeito, estendendo o escopo da aplicação dos FCM e propondo um novo tipo de DCN.

\section{Construção da DCN}

De acordo com a literatura, os FCM melhoraram os $\mathrm{CM}$, descrevendo a força das relações causais através de números Fuzzy, ou seja, ponderações das opiniões dos especialistas de acordo com nível de conhecimento ou domínio do problema por meio de funções de pertinência Fuzzy. As DCN melhoram os FMC, permitindo que cada conceito selecione sua relação de causalidade com os outros conceitos de acordo com as exigências do sistema. Essas relações assumem valores que podem ser retirados de um conjunto binário, de um conjunto Fuzzy, ou de uma 
função contínua ou trivalente. ADCN proposta nesse trabalho extrapola esse comportamento na medida em que introduz procedimentos para determinar como as causas produzem efeitos de forma sazonal através do disparo de regras que identificam a ocorrência simultânea de vários fatores (causa) ou a partir de funções matemáticas em que o tempo é uma variável independente. Contudo, o efeito da causa também pode ser variante no tempo. Deste modo, as DCN aqui propostas podem ser consideradas modelos causais com habilidades dinâmicas em que as relações de causalidade e os efeitos são variáveis dependentes do tempo. Um exemplo de estratégia de time de futebol de robôs será apresentada para exemplificar a dificuldade de tratamento de tempo no FCM e uma possível solução através da aplicação da DCN.

A DCN proposta se comporta de acordo com a complexidade do problema abordado. Para as decisões de mais baixo nível, em que se admitem apenas relações de causa-efeito positiva, negativa e neutra entre conceitos, a DCN se comporta como um mapa cognitivo simples. Para decisões com vários fatores implicados, a DCN se comporta como um FCM, em que as relações causais e conceitos são números $F u z z y$, porém considera-se que todas as causalidades ocorrem de modo simultâneo. Para os sistemas dinâmicos mais complexos, a DCN é capaz de alterar dinamicamente os valores de suas relações conceitos, além de inserir e/ou eliminar novos conceitos e relações quando necessário, constituindo-se assim em um modelo sofisticado de um sistema dinâmico não-linear multivariável.

De um modo geral, todos os trabalhos citados contribuíram no desenvolvimento, porém, podemse citar cinco trabalhos que tiveram maior influência na arquitetura DCN. O trabalho Stylios, Groumpos e Georgopoulos (1999) apresentou a necessidade de ajuste no FCM por meio de dados de operação (conhecimento quantitativo) após aquisição do conhecimento qualitativo dos especialistas. Esse trabalho contribuiu para verificar as dificuldades de se construir modelos dinâmicos utilizando um
FCM clássico (KOSKO, 1986). Já o trabalho de Papageorgiou, Stylios e Groumpos (2003) inspirou a construção da estrutura dividida em dois níveis de conhecimento e a inclusão de conceitos de seleção e fator para tomadas de decisões. Os trabalhos de Carvalho, em especial (CARVALHO, 2001) contribuíram na inclusão de conceitos de variação, inclusão de relações do tipo Fuzzy e no tratamento dinâmico da informação.

No contexto de aquisição e tratamento de dados, trabalhos que utilizam a DCN como aproximador de função, em especial como preditor, também são conhecidos na literatura, tais como demonstrado na pesquisa realizada por Arthi, Papageorgiou e Tamilarasi (2011).

Finalmente, os trabalhos de Miao et al. (2010) contribuíram na inclusão de relações matemáticas temporais, na construção de inferências não monotônicas e na conceituação da DCN como uma evolução dos mapas cognitivos Fuzzy. Neste contexto, uma proposta de uma arquitetura cognitiva inicialmente modelada de forma estruturada, baseada em CM e principalmente em FCM é então expandida pela inclusão de novos tipos de conceitos e relações baseados em regras do tipo antecedenteconsequente e não apenas aquelas do tipo causaefeito, estendendo o escopo da aplicação dos FCM e propondo um novo tipo de DCN.

\section{Modelo de uma rede cognitiva dinâmica}

A DCN proposta nesse artigo é uma evolução dos mapas cognitivos Fuzzy e incorpora novos tipos de relações e conceitos que as tornam mais aptas a modelagem de sistemas dinâmicos complexos. Para tratamento de incertezas, não-linearidades e inclusão de um caráter temporal nas relações entre conceitos utiliza-se uma relação causal cujo valor é variante no tempo. O valor desta relação pode ser obtido a partir de uma função trivalente, ou mesmo de uma função dinâmica no domínio de Laplace como proposto por Miao et al. (2001), ou como resultado da execução de uma base de regras ou 
mesmo de um sistema Fuzzy. Esse tipo de relação, denominado relação de seleção, também confere às DCN a capacidade de raciocínio não monotônico.

A DCN proposta possui os seguintes tipos de conceitos e relação:

- conceito de entrada e saída de dados: fazem a interface com os dispositivos de entrada e saída. Esses conceitos têm suas entradas e saídas normalizadas;

- conceito de nível: é utilizado quando se pode utilizar o valor absoluto de uma variável de um processo, e auxilia na representação da evolução dinâmica de um modelo físico;

- conceito variação: representa a equação da diferença em primeira ordem de uma determinada grandeza em um intervalo de tempo conhecido. Deste modo, esse conceito pode, por exemplo, junto com conceito de nível auxiliar na construção de uma base de regras, para tomadas de decisões dinâmicas em um processo considerando o valor de uma variável e sua variação em um determinado contexto;

- conceito de decisão ou relação: são utilizados quando se necessita mudar e/ou atualizar conceitos (variáveis) em uma $\mathrm{DCN}$, porém não ocorre interação com outros conceitos. Esses conceitos são inseridos na DCN através de relações de seleção;

- conceito de memória: responsável por armazenar um valor atrasado no tempo. A utilização deste tipo de conceito na DCN insere no modelo cognitivo uma capacidade de decisão que considera situações passadas do sistema;

- relação de fator: insere uma operação de multiplicação normalmente ponderada no conceito que está conectada na saída da DCN. Essa relação é ativada por regras do tipo declaração condicional;

- relação causal pura: são as relações que estabelecem a causa-efeito entre os conceitos de um mapa cognitivo como proposto por Axerold (1976) e Kosko (1986). As relações causais são calculadas através de uma matriz $\mathrm{W}$ constante;

- relação causal variante no tempo: estabelece uma causa-efeito entre os conceitos de um mapa cognitivo, dirigida por uma função do tempo como proposto por Miao et al. (2001, 2010). Essas relações causais são calculadas por meio de uma matriz W em função do tempo;

- relação Fuzzy: permite a construção de tomadas de decisões do tipo não monotônica, é caracterizada quando se tem a influência direta de dois conceitos em um terceiro. A matriz W resultante também é variante no tempo de acordo com o resultado da execução de um sistema Fuzzy;

- relação de seleção: permite representar a ocorrência de eventos e identificar quais são as relações de causa-efeito válidas em um dado instante de tempo. Essa relação é ativada através da execução de uma ou mais regras do tipo "seentão", que tem seu disparo e duração de acordo com o fenômeno modelado. Essas relações são naturalmente variantes no tempo.

\section{Algoritmo para construção de uma DCN}

A construção de uma DCN (Tabela 1) segue inicialmente os mesmos passos utilizados no desenvolvimento de mapas cognitivos e mapas cognitivos Fuzzy. Entretanto, para a construção de tomadas de decisões estratégicas, devem-se acrescer mais etapas ou passos no desenvolvimento da DCN.

A utilização do algoritmo detalhado na Tabela 1, para modelagem de sistemas dinâmicos, pode sofrer pequenas alterações, dependendo do tipo de problema que se quer modelar (controle supervisório, robótica, mundo virtual, tomada de decisão, etc.). Por exemplo, pode não ser necessário o uso de relações Fuzzy ou de conceitos do tipo variação, ou ainda, pode não ser necessária uma etapa de treinamento ou de aprendizado, pois os 
algoritmos utilizados em cada uma das etapas podem ser diferentes. Um dos passos mais importantes na qualidade do modelo obtido está na realização das etapas 1 e 2 do algoritmo exposto na Tabela 1. De fato, a abstração do conhecimento necessário para a realização dos passos não é uma simples tarefa e, se alguma informação relevante ao comportamento do sistema não for modelada, certamente prejudicará as etapas sequentes na construção da DCN.

Tabela 1 - Construção de uma DCN.

Etapa 1: Identificação dos conceitos e suas funções (entrada, saída, fator e seleção), as suas interligações, e/ou seleção das relações entre conceitos, determinando sua natureza causal (positiva, negativa, neutra) e seus tipos (Fuzzy, seleção, fator, causal).

Etapa 2: Aquisição de dados inicial, através da opinião ponderada de especialistas e/ou análise de um modelo matemático, ou análise de dados e/ou comportamento dinâmico do sistema.

Etapa 3: Apresentação de dados sobre as opiniões de peritos para um sistema de inferência Fuzzy que tem como resultado os valores de pesos da DCN.

Etapa 4: A construção da base de regras Fuzzy que tem como inferência os valores de pesos da DCN para as relações Fuzzy, caso esse tipo de relação seja implementada.

Etapa 5: Tratamento da informação, adaptação e otimização da DCN, ajustando as suas respostas para a saída desejada. Caso necessário, são empregados algoritmos de aprendizagem por reforço para refinamento dinâmico do modelo.

Etapa 6: Construção da base de regras dos conceitos e relações de seleção, e, caso seja implementada, construção das regras de aprendizado por reforço.

Etapa 7: Validação do modelo DCN.

Fonte: Mendonça (2011).

As etapas 1, 2 e 3 são semelhantes ao desenvolvimento de um FCM clássico, ou seja, DCNi (DCN inicial) (Identificação, aquisição e números Fuzzy). A etapa 4 (relação Fuzzy) é específica de uma DCNi, porém modela causa e efeito de modo contínuo e é opcional. A etapa 5 é a adaptação (FCM, DCNi ou DCN 1 [DCN na fase de evolução 1]). A etapa 6 é a inclusão base de regras (nível estratégico) $\mathrm{DCN}_{\mathrm{f}}$ (DCN final) ou $\mathrm{DCN}_{2}$ (DCN na fase de evolução 2). Finalmente, a etapa 7 faz a validação do modelo e pode ser usada a cada nível de construção da DCN. Inicialmente, devese validar a DCNi (causa-efeito). Posteriormente, pode-se validar a $\mathrm{DCN}_{\mathrm{f}}$ com a inclusão das ações de planejamento (base de regras e eventos) e adaptação dinâmica do modelo de forma opcional.

A arquitetura DCN, conforme mostrada na Figura 1, de um modo geral apresenta dois blocos extremos que representam as interfaces de entrada e saída e um bloco inferior que representa o motor de inferência e/ou tomada de decisão.

Os dois blocos superiores representam a base de regras e relações para tratamento do conhecimento na forma dinâmica e de eventos. Essa representação em blocos auxilia no entendimento do funcionamento da arquitetura como um todo. Deste modo, as aplicações que empreguem DCN terão representações gráficas semelhantes, basicamente com diferenças nas variáveis de entrada e saída. $\mathrm{Na}$ prática, esses blocos darão origem a conceitos e relações como, por exemplo, os blocos de comunicação com o ambiente em que o modelo será inserido são feitos através de conceitos de entrada e saída da DCN. O bloco relações causais e Fuzzy representa as tomadas de decisões contínuas, suas respectivas relações e os conceitos conectados. O bloco base de regras linguísticas representa as outras relações, em especial, às relações e conceitos de seleção e fator com suas respectivas regram que modelam as ações orientadas pela ocorrência de eventos. Essa arquitetura explora a capacidade de aquisição de conhecimento estruturado, com diferentes fontes de conhecimento. Essas fontes podem ou não ter a mesma origem, o que permite a construção de tomadas de decisões de baixo nível (relações causais) e estratégicas por um especialista 
trabalhando de modo simultâneo ou não white board (COPPIN, 2010).

Figura 1 - Arquitetura DCN.

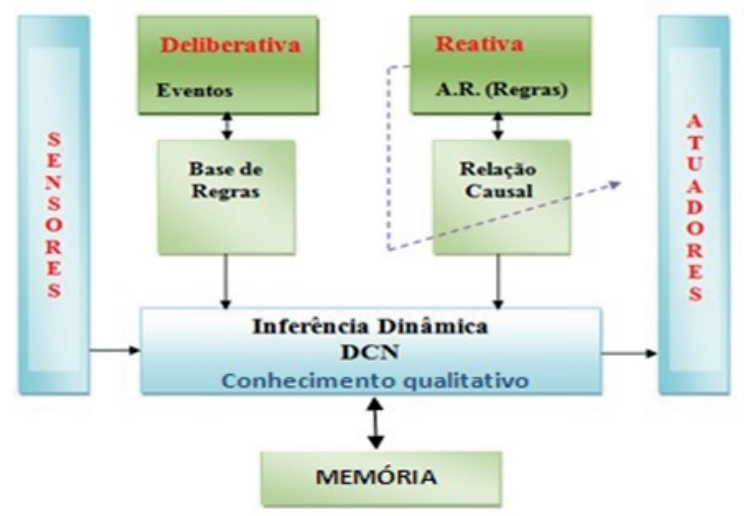

Fonte: Mendonça (2011).

\section{DCN Aplicada a Tomadas de Decisões Dinâmicas}

O algoritmo proposto e a arquitetura de representação de conhecimento proposta na seção anterior serão validados num exemplo simples de futebol de robôs. De acordo com a literatura, o desenvolvimento de estratégias de times de futebol de robôs é um problema dinâmico de tomada de decisão fortemente influenciado por mudanças no ambiente, sendo por isso um exemplo de aplicação bastante utilizado para validação de sistemas inteligentes (COSTA; PEGORARO, 2000).

Diversos módulos compõem o sistema de um time de futebol robôs, os principais são: visão, estratégia e controle. O módulo de estratégia define, para cada robô, uma posição alvo-posição a ser atingida no campo, as respectivas velocidades e orientação associadas, para que esses dados possam ser traduzidos em termos de velocidades e sentido de rotação dos motores que comandam as rodas dos robôs. A definição da posição alvo depende do estado do jogo e dos comportamentos associados a cada robô.
A estratégia empregada para construção de uma DCN é baseada no trabalho de Costa e Pegoraro (2000) que modela o problema através de uma máquina de estados capaz de tomar as decisões do time (Figura 2). A estratégia a ser modelada é desenvolvida para dois jogadores de linha e um goleiro e é implementada através de ações simples tais como ir a determinado ponto do campo ou seguir a bola. A estratégia global é formada por uma composição dessas estratégias mais simples com base na situação do jogo. As funções de cada jogador variam de acordo com a sua posição em campo. O goleiro deve permanecer na área do gol e tem como objetivo evitar que a bola passe da linha que limita o gol se locomovendo lateralmente. O jogador de linha pode funcionar como zagueiro se estiver posicionado no seu campo defensivo ou como atacante se estiver no campo adversário. O zagueiro tem a função de evitar que algum jogador adversário aproxime-se do gol com a bola sob controle. Já o atacante tem a função de recuperar a bola e conduzi-la até o gol do adversário, tomando decisões de qual ângulo e com que intensidade chutará a bola em direção ao gol.

Figura 2 - DCN - Estratégia de futebol de robôs.

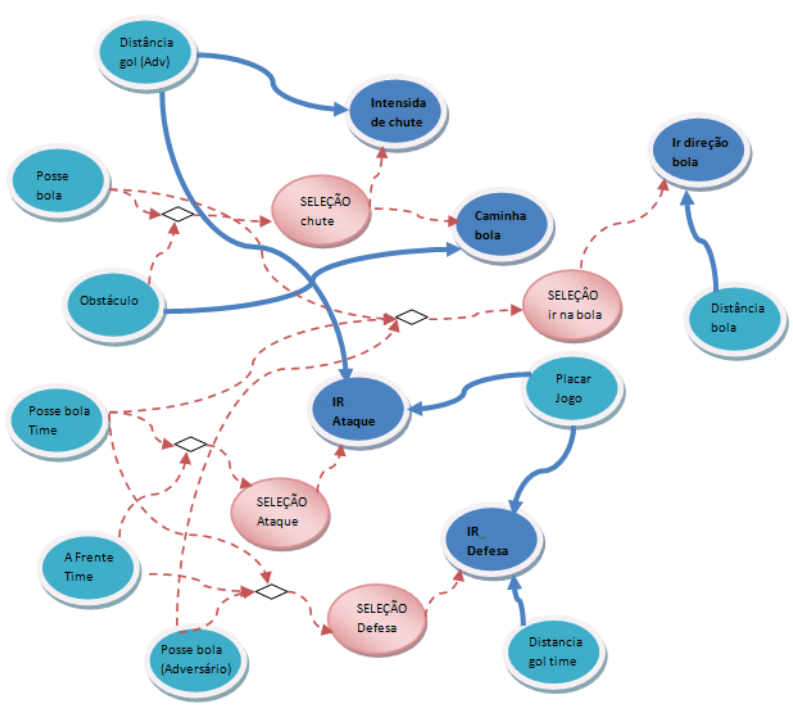

Fonte: Mendonça (2011). 
Por simplicidade de funcionalidades, quando um jogador ataca o outro defende. Deve-se observar que um FCM clássico não se aplica a solução do problema, devido existir ações contraditórias, ou seja, ou o jogador chuta ou caminha com a bola, não podendo ocorrer as duas ações de modo simultâneo.

De acordo com a estratégia apresentada, a heurística de controle para um mesmo jogador, que pode atuar como atacante ou como zagueiro, corresponde passo a passo à seleção de uma das ações listadas a seguir:

- chutar em direção ao gol;

- caminhar junto com a bola (bola com o jogador);

- ir para a posição de ataque;

- ir para a posição de defesa;

- ir ao encontro da bola.

A DCN então permite tomada de decisões por disparo de eventos, ou seja, caso o jogador tenha posse de bola e não estiver muito longe do gol, poderá chutar. Caso não tenha posse de bola, pode então ir à direção do adversário, tomadas de decisões que não poderia ser modelada com um FCM clássico. As duas subseções abordam, de modo resumido, duas diferentes áreas de conhecimento com resultados da aplicação da DCN objetivando mostrar a versatilidade da ferramenta.

\section{Exemplos de aplicações em controle de processos}

A área de controle de processos, em especial sistemas supervisórios, utilizam-se de tomadas de decisões de operadores. O conhecimento desses especialistas pode ser modelado inicialmente através de mapas cognitivos com a finalidade de se determinar as interconexões das variáveis envolvidas. Contudo, a modelagem cognitiva de um sistema supervisório em um nível mais alto de complexidade passa de um FCM para uma DCN, haja vista que o controle supervisório necessita de tomadas de tempo dinâmicas, sendo a principal restrição do FCM.
O controle de nível descrito a seguir é um estudo de caso bastante utilizado na literatura (GLYKAS, 2010), (STYLIOS; GROUMPOS; GEORGOPOULOS, 1999). Portanto, na Figura 3 é esquematizado um tanque com duas válvulas de entrada para diferentes líquidos, um misturador, uma válvula de saída para retirada do líquido produzido pela mistura e um medidor de massa específica que mede a qualidade do líquido produzido.

Figura 3 - Tanque misturador

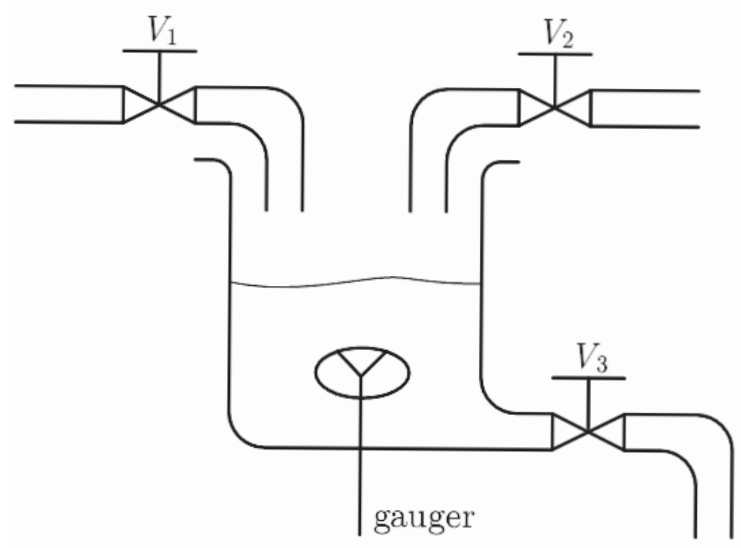

Fonte: Adaptada de Stylios, Groumpos e Georgopoulos (1999).

As válvulas $\left(\mathrm{V}_{1}\right)$ e $\left(\mathrm{V}_{2}\right)$ inserem dois diferentes líquidos no tanque. Durante a reação dos dois líquidos, é produzido um novo líquido caracterizado por seu valor de massa específica. A válvula $\left(\mathrm{V}_{3}\right)$ esvazia o tanque de acordo com uma campanha.

O desenvolvimento da DCN é realizado através de duas etapas distintas. Primeiramente a DCN é desenvolvida com um FCM clássico onde são identificados os conceitos e as relações causais, os conceitos podem ser variáveis e/ou ações de controle. Nesse caso, como já sugerido, os conceitos foram os cinco citados (conceito de entrada e saída de dados, conceito de nível, conceito variação, conceito de decisão ou relação e conceito de memória) e a heurística de controle é a seguinte: se o nível de volume ou peso da mistura aumentar, as válvulas de entrada são fechadas, desse modo é possível atribuir causalidade negativa 
entre os conceitos de nível e de saída de dados. Já a relação entre a vazão da válvula de saída define uma causalidade positiva, ou seja, quando ocorre um aumento de vazão de saída. De acordo com uma campanha de processo desejada, as válvulas de entrada também aumentam proporcionalmente. O ajuste inicial é feito por meio de um algoritmo baseado na heurística de otimização baseada no método Simulated Annealing (GHAZANFARI et al., 2007), na qual uma solução inicial é lançada como um chute inicial e depois soluções com certo grau de aleatoriedade é sistematicamente testado até que o sistema obtenha a resposta desejada. $\mathrm{Na}$ Figura 4 é demonstrado o esquema gráfico de um controlador DCN.

Figura 4 - Controlador DCN para o misturador industrial.

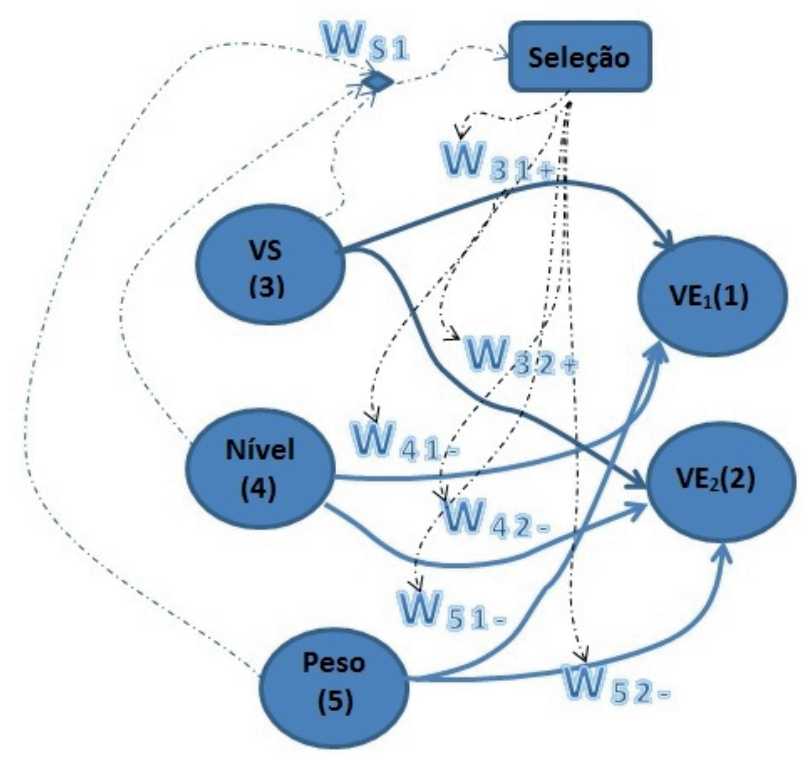

Fonte: Fonte própria.

A segunda etapa de desenvolvimento da DCN é responsável pela sintonia ou refinamento do modelo para resposta dinâmica do controlador. Nesse caso, quando ocorre uma mudança de setpoint na campanha de saída, os pesos das relações causais são sintonizados. Para realizar essa função foram inclusos, no modelo cognitivo, um novo tipo de conceito e de relação. A relação de seleção atribui regras orientadas a eventos, que nesse caso foi relativa à mudança de set-point e níveis do peso e do volume da mistura líquida. A DCN utiliza o conceito de seleção pra chavear o conjunto de relações causais de acordo com a base de regras da relação de seleção. A DCN funciona de modo semelhante a um DT-FCM (decision tree-FCM) (PAPAGEORGIOU; STYLIOS; GROUMPOS, 2006), de outro modo, a DCN se assemelha a uma ferramenta híbrida entre um FCM e uma máquina de estados cíclica temporal, chaveada por disparo de eventos, semelhante ao trabalho de Acampora e Loia (2011). Na Figura 5, o resultado do controlador é mostrado.

$\mathrm{Na}$ Figura 6, é mostrada uma das saídas das variáveis controladas pela DCN (o peso da mistura líquida dentro de uma faixa de controle pré-estabelecida). Observa-se que pela baixa complexidade computacional do algoritmo de controle da DCN, o projeto encontra-se em fase de desenvolvimento em um sistema embarcado na plataforma Arduino.

Figura 5 - Resultado do controlador DCN

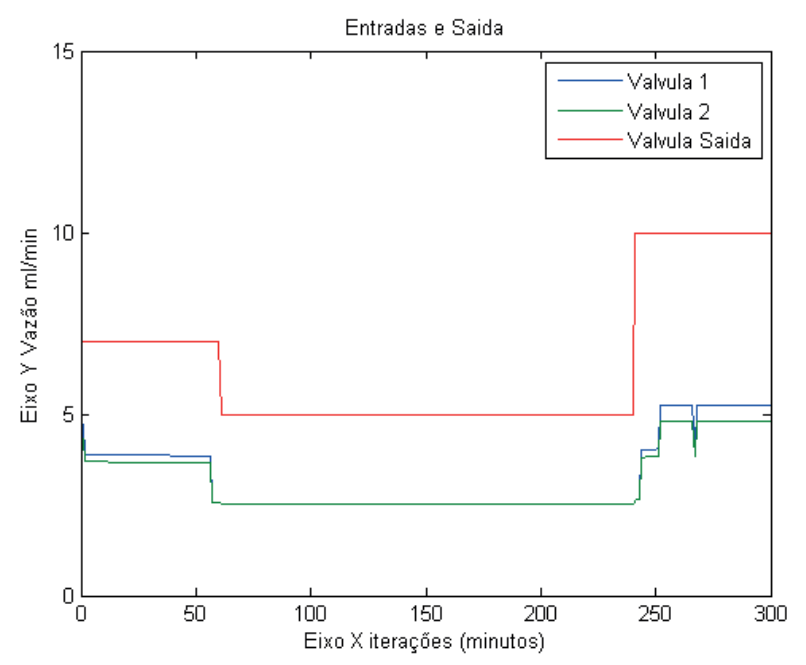

Fonte: Fonte própria.

Já na Figura 7, é mostrado o modelo final de uma DCN aplicada a controle supervisório. Nessa DCN, relações de seleção Fuzzy e causais 
associadas a conceitos clássicos e fator, compõem a lógica que envia set-points aos controladores PID (Controlador Proporcional, Integral e Derivativo) de um fermentador, por exemplo.

Figura 6 - Saída da variável peso do Tanque do Controlador DCN

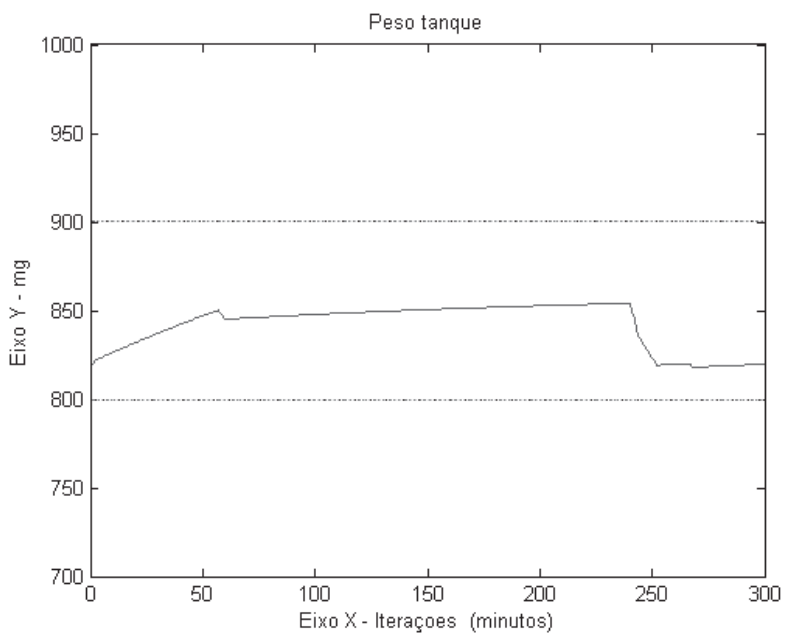

Fonte: Fonte própria.

A DCN possibilita aumentar a eficiência de controle através de disparo de regras e inclusão de funcionalidades como, por exemplo, mudar a campanha caso o substrato esteja com valores próximos de zero, significando que a reação química está na iminência de acabar.

Na Figura 8, visualiza-se o gráfico com as variáveis do processo. Na segunda parte da figura, quando o substrato chega a níveis próximos a zero, a campanha é alterada, conforme pode ser visto em comparação à primeira parte da figura. Na terceira a quarta parte da figura, observa-se respectivamente a biomassa e o volume.
Figura 7 - DCN final

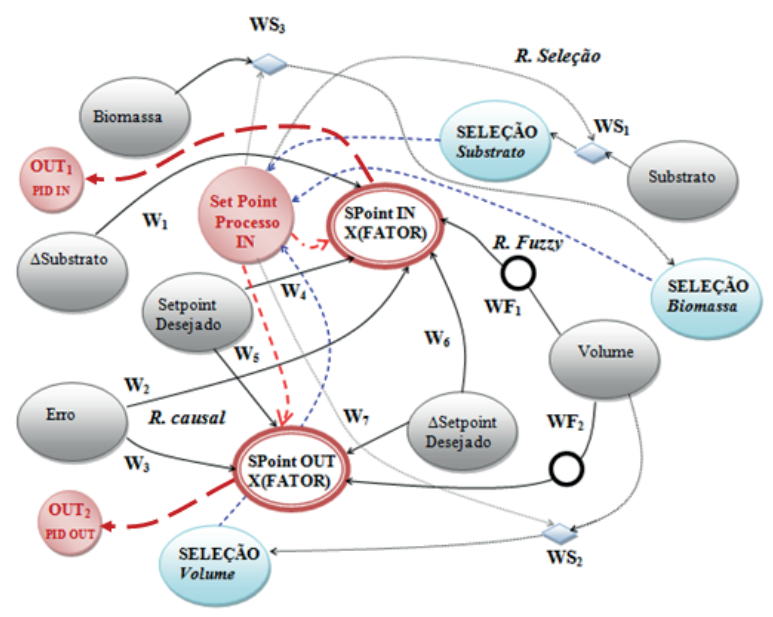

Fonte: Mendonça (2011).

Figura 8 - Controle supervisório (DCN+PID)
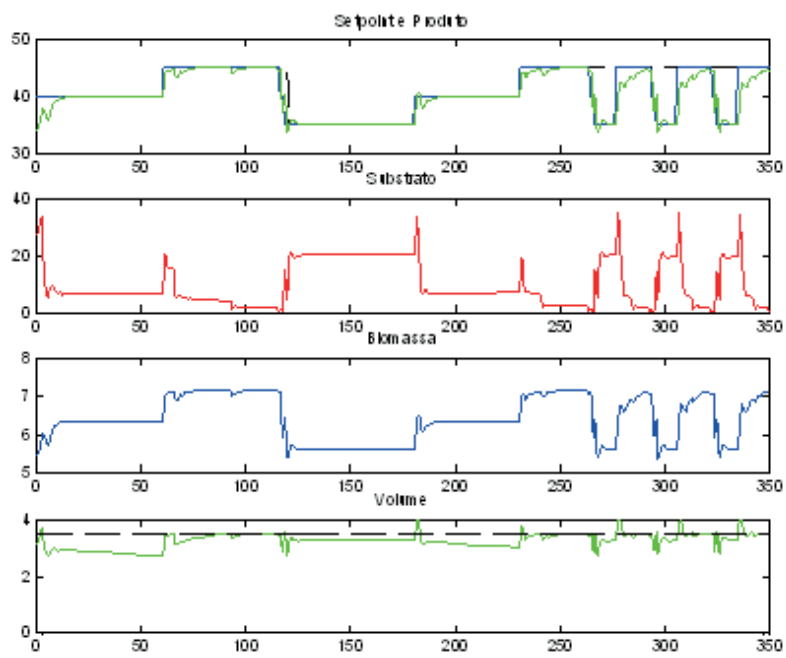

Fonte: Mendonça (2011).

No trabalho de Mendonça, Arruda e Neves Junior (2011), detalhes são demonstrados de um processo de fermentação alcoólica, com desenvolvimento e apresentação de resultados, utilizando uma DCN para o controle do processo.

\section{Exemplo de aplicação para navegação robótica}

Robôs móveis é um assunto atraente entre pesquisadores. Neste sentido, pode ser um terreno útil comum para comparar diferentes soluções 
para os mesmos problemas. Pesquisas utilizando sistemas computacionais inteligentes têm sido frequentes na construção de sistemas de navegação autônomos, em especial em aplicações com pouco ou nenhum conhecimento do ambiente (COSTA; GOUVEA, 2010).

Neste contexto, uma proposta de um sistema de navegação através de aplicação da DCN pode ser vista na Figura 9. Para a construção de um controlador de um sistema de desvio de obstáculos são utilizados conceitos de entrada e saída para a comunicação com os sensores e atuadores. Os conceitos de memória como um registro do movimento imediatamente anterior objetiva manter uma tendência de movimento em tempo de navegação.

Figura 9 - DCN navegação autônoma (coleta alvos e desvio obstáculos)

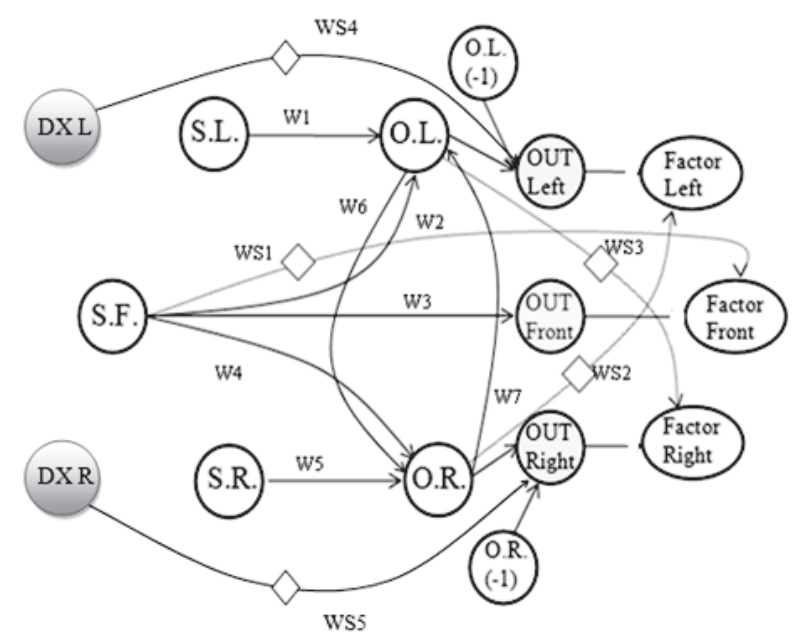

Fonte: Mendonça (2011).

Para entender a ocorrência desse efeito "zigzag”, relações do tipo seleção (WS1, WS2 E WS3) são modeladas por uma base de regras. No trabalho de Mendonça, Arruda e Neves Junior (2010) é então formalizada o ED-FCM (Event Driven-FCM), essa técnica foi precursora desta proposta de navegação autônoma baseada em DCN e compara resultados iniciais (desvio de obstáculos) com controlador Fuzzy. Já na pesquisa realizada por Mendonça et al. (2011), é apresentada a arquitetura da DCN baseada na arquitetura de subsunção sintonizada dinamicamente por algoritmos de aprendizagem por reforço. Nesse mesmo trabalho são apresentados mais resultados em cenários diferentes. Na Figura 10, é apresentada a memória de posições (rastro) da navegação do agente móvel através de um cenário praticamente desconhecido. Em outras palavras, o controlador DCN navega com praticamente nenhum conhecimento do cenário (somente a posição dos alvos representados por triângulos) e nenhum conhecimento das posições dos obstáculos.

Os resultados mostram que o agente cumpre seus objetivos devido à coleta dos alvos “ $\boldsymbol{\Delta}$ " e do desvio dos obstáculos "+" estáticos no cenário, entretanto, um dos obstáculos aparece sempre de forma inesperada (obstáculo surpresa). Finalizando, o agente móvel chega a uma região final desejada sem nenhuma colisão e com alvos coletados.

Figura 10 - Memória de posição móvel (rastro)

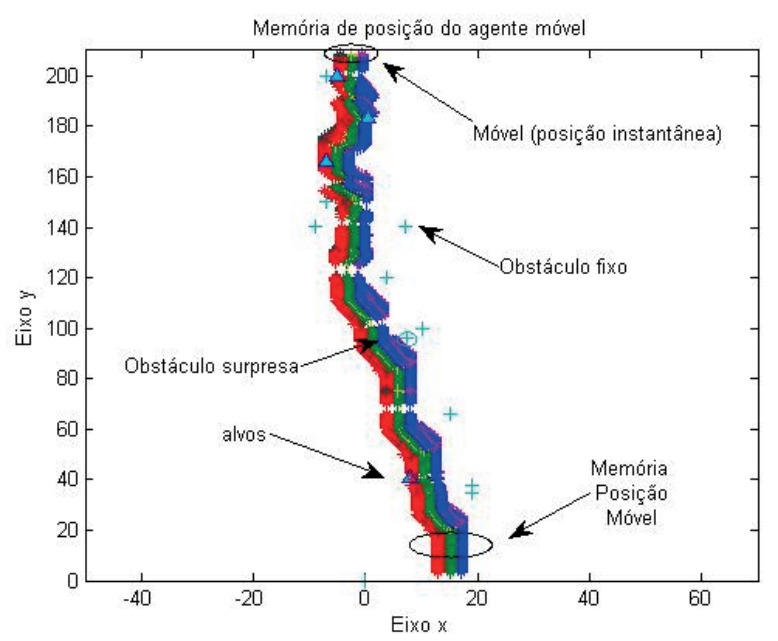

Fonte: Fonte própria.

Na Figura 11, é mostrada a DCN implementada em uma base robótica (Dr Robot X-80) na sua versão inicial, somente com a funcionalidade de desvio de obstáculos (MENDONÇA, ARRUDA e NEVES, 2010). 
Figura 11 - Imagem da trajetória e do robô móvel (desvio de obstáculos).

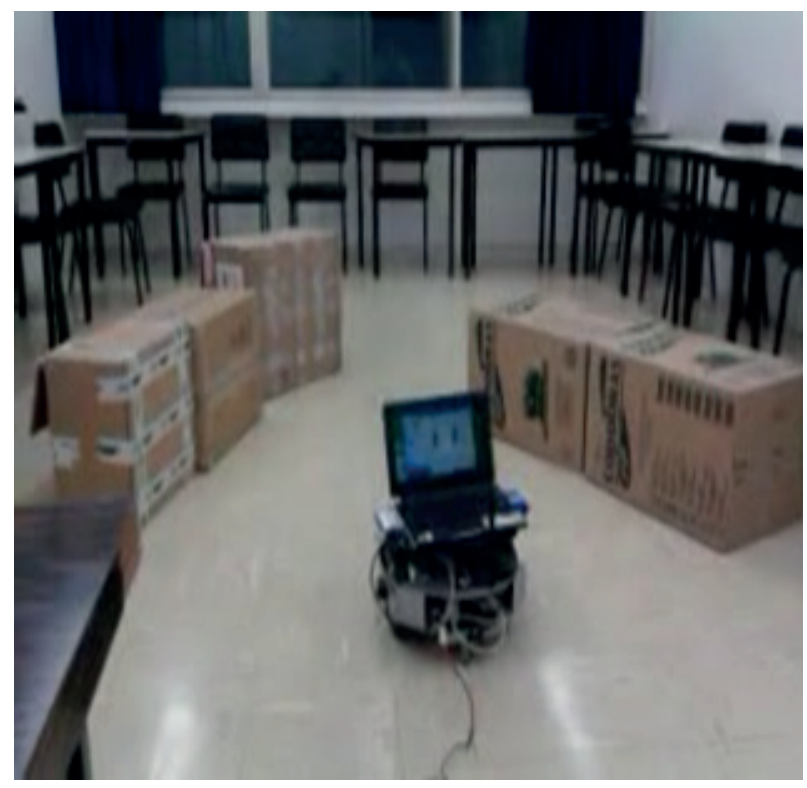

Fonte: Mendonça (2011).

\section{Conclusões}

Esse artigo apresentou uma contribuição para o desenvolvimento de sistemas inteligentes através de uma metodologia baseada em redes dinâmicas cognitivas. A arquitetura desenvolvida permite a tomada de decisões dinâmicas através do mapeamento de duas camadas distintas que modelam diferentes tipos de conhecimento. As referidas camadas cooperam e contribuem para o sucesso da aplicação, eventualmente sendo necessário utilizar métodos de treinamento (Redes Neurais Artificiais, Algoritmos Genéticos, Particle Swarm Optimization, entre outros) para calibração e sintonia off-line do modelo, podendo-se também empregar técnicas algorítmicas de aprendizagem por reforço para aumentar a capacidade de adaptação dinâmica do modelo.

Esta arquitetura não é restrita a apenas as aplicações apresentadas neste trabalho, podendo também ser aplicada na construção de qualquer tipo de sistema em que se precise modelar a tomada de decisão on-line a partir de eventos dinâmicos.
A arquitetura baseada em DCN constitui uma ferramenta flexível e robusta, capaz de modelar a imprecisão e incertezas dos sistemas dinâmicos de um modo formal, através de uma modelagem matemática baseada em grafos. Uma das principais vantagens da abordagem proposta é que a aquisição e a representação do conhecimento são facilitadas pelo uso de mapas cognitivos, que por gerarem códigos simples de software podem serem facilmente embarcados em hardware dedicado.

Futuros trabalhos endereçam aplicações com robôs reais em navegação e estratégia de times de futebol, novas funcionalidades no controle supervisório do fermentador e a exploração em outras áreas de conhecimento, como por exemplo, gerenciamento e controle de tráfego. Enfim, na arquitetura proposta pode-se utilizar técnicas de soft-computing (Algoritmos Genéticos, Ant Colony Optimization, Particle Swarm Optimization, Simulated Annealing, entre outros), ou até mesmo modelos híbridos baseados no aprendizado de Hebb (PAPAGEORGIOU, 2012) para soluções off-line na construção de redes cognitivas dinâmicas com maior número de conceitos e sua maior integração por meio de protocolos de compartilhamento de dados oriundos da teoria de agentes inteligentes, em especial, focando em arquiteturas cognitivas autônomas baseadas em sistemas bioinspirados, como aplicações em robótica coletiva (Swarm Robotics).

\section{Referências}

ACAMPORA, G.; LOIA, V. On the temporal granularity in Fuzzy cognitive maps. IEEE Transactions on Fuzzy Systems, New York, v. 19, n. 6, p. 1040-1057, 2011.

ARTHI, K.; PAPAGEORGIOU, E. I.; TAMILARASI, A. Analyzing the performance of Fuzzy cognitive maps with non linear hebbian learning algorithm in predicting autistic disorder. Expert Systems with Applications, Philadelphia, v. 38, p. 1282-1292, 2011.

AXELROD, R. Structure of decision: the cognitive maps of political elites. New Jersey: Princeton University Press, 1976. 
CARVALHO, J. P. B. Mapas cognitivos baseados em regras difusas: modelação e simulação da dinâmica de sistemas qualitativos. 2001. Dissertação (Doutorado em Engenharia Electrotécnica e de Computadores) Universidade Técnica de Lisboa, Lisboa.

COPPIN, B. Inteligência artificial. Rio de Janeiro: Livros Técnicos e Científicos, 2010.

COSTA, A. H. R.; PEGORARO, R. Construindo robôs autônomos para partidas de futebol: o time guaraná. $S B A$ Controle \& Automação, Campinas, v. 11, n. 3, p. 141149, 2000.

COSTA, E. D. S.; GOUVEA, M. M. Autonomous navigation in dynamic environments with reinforcement learning and heuristic, machine. In: INTERNATIONAL CONFERENCE ON MACHINE LEARNING AND APPLICATIONS (ICMLA), 9., 2010, Washington. Proceedings... Washington: ICMLA, 2010. p. 37-42.

DICKERSON, J. A.; KOSKO, B. Virtual worlds as Fuzzy cognitive maps. Presence, Trier, v. 3, n. 2, p. 173189, 1994.

GHAZANFARI, M.; ALIZADEH, S.; JAFARI, M. Using Fuzzy expert system for solving Fuzzy system dynamics. In: EURO ASIA-ICT CONFERENCE (ADVANCES IN INFORMATION AND COMMUNICATION TECHNOLOGY), 2002, Shiraz. Proceedings... Shiraz, 2002.

GHAZANFARI, M.; ALIZADEH, S.; FATHIAN, M.; KOULOURIOTIS, D. E. Comparing simulated annealing and genetic algorithm in learning FCM. Applied Mathematics and Computation, Carlton Court, v. 192, n. 1, p. 56-68, 2007.

GLYKAS, M. Fuzzy cognitive maps: advances in theory, methodologies, tools and applications. Berlin: Springer, 2010.

HAYKIN, S. Redes neurais, princípios e prática. 2. ed. São Paulo: Bookman, 2000.

HUANG, Y. C.; WANG, X. Z. Application of Fuzzy causal networks to waste water treatment plants. Chemical Engineering Science, Brisbane, v. 54, n. 13/14, p. 2731-2738, 1999.

KOUlOURIOTIS, D. E., DIAKOULAKIS, I. E.; EMIRIS, D. M. Learning Fuzzy cognitive maps using evolution strategies: a novel schema for modeling and simulating high-level behavior. In: IEEE CONGRESS ON EVOLUTIONARY COMPUTATION (CEC2001), 2001, Seoul. Proceedings... Seoul, 2001. p. 364-371.
KOULOURIOTIS, D. E; DIAKOULAKIS I. E.; EMIRIS, D. M.; ZOPOUNIDIS, C. D. Development of dynamic cognitive networks as complex systems approximators: validation in financial time series. Applied Soft Computing, Cranfield, v. 5, n. 2, p. 157-179, 2005.

KOSKO, B. Fuzzy cognitive maps. International Journal Man-Machine Studies, Milton Keynes, v. 24, n. 1, p. 6575, 1986.

KOTTAS,T.L.;BOUTALLIS,Y.S.;CHRISTODOULOU, M. A. Fuzzy cognitive network: a general framework. Intelligent Decision Technologies, Baltimore, v. 1, n. 4, p. 183-196, 2007.

LEE, K. C.; LEE, S. A. Cognitive map simulation approach to adjusting the design factors of the electronic commerce web sites. Expert Systems with Applications, Philadelphia, v. 24, n. 1, p. 1-11, 2003.

MENDONÇA,M.Uma Contribuição ao Desenvolvimento de Sistemas Inteligentes Utilizando Redes Cognitivas Dinâmicas. Tese (Doutorado), Universidade Tecnológica Federal do Paraná, 2011.

MENDONÇA, M.; ARRUDA, L. V. R.; NEVES, F. J. Qualitative autonomous navigation system employing event Drive-Fuzzy cognitive maps and Fuzzy logic. In: CONGRESSO BRASILEIRO DE AUTOMÁTICA, 2010, Bonito. Anais... Bonito, 2010. p. 1-6.

MENDONÇA, M.; ARRUDA, L. V. R.; NEVES JUNIOR, F. Redes dinâmicas cognitivas aplicadas no controle supervisório de um fermentador. Sociedade Brasileira de Automática, Campinas, v. 22, n. 4, jul./ago. 2011.

MENDONÇA, M.; ANGÉLICO, B. A.; ARRUDA, L. V.; NEVES, F. Arquitetura de subsunção baseada em redes cognitivas dinâmicas com aplicação em navegação autônoma. In: SIMPÓSIO BRASILEIRO DE AUTOMAÇÃO INTELIGENTE, 10,, 2011, São João del Rei. Anais... São João del Rei: SBAI, 2011.

MIAO, Y.; LIU, Z. Q.; SIEW, C. K.; MIAO, C. Y. Dynamical cognitive network: an extension of Fuzzy cognitive. IEEE Transactions on Fuzzy Systems, New York, v. 9, n. 5, p. 760-770, 2001.

MIAO, Y. Z. Q.; LIU, C. K.; SIEW, C. K.; MIAO, C. Y. Transformation of cognitive maps. IEEE Transactions on Fuzzy Systems, New York, v. 18, n. 1, p. 114-124, 2010.

PAPAGEORGIOU, E.; STYLIOS, C. D.; GROUMPOS, P. P. Decision making in external beam radiation therapy based on Fuzzy cognitive maps. In: INTERNATIONAL IEEE SYMPOSIUM ON INTELLIGENT SYSTEMS, 2003, Varma. Proceedings... Varna, Sept. 2003. p. 312317. 
PAPAGEORGIOU, E.; STYLIOS, C.; GROUMPOS, P. A. Combined Fuzzy cognitive map and decision trees model for medical decision making. Conference proceedings : ... Annual International Conference of the IEEE Engineering in Medicine and Biology Society, Piscataway, v. 1, p. 6117-6120, 2006.

PAPAGEORGIOU, E.; STYLIOS, C.; GROUMPOS, P. Novel for supporting medical decision making of different data types based on Fuzzy Cognitive Map Framework. ANNUAL INTERNATIONAL CONFERENCE OF THE IEEE EMBS CITÉ INTERNATIONALE, 29., 2007, Lyon. Proceedings... Lyon, Aug. 2007.

PAPAGEORGIOU, E. Learning algorithms for Fuzzy cognitive maps. IEEE Transactions on Systems and Cybernetics. part C: Applications and Reviews, Lamia, v. 42 , n. 2, p. 150-163, 2012.
PERUSICH, K. Fuzzy cognitive maps for policy analysis. In: INTERNATIONAL SYMPOSIUM ON TECHNOLOGY AND SOCIETY TECHNICAL EXPERTISE AND PUBLIC DECISIONS, 1996, Princeton. Proceedings... Princeton: IEEE, 1996. p. 369373.

STYLIOS,C.D.; GROUMPOS,P.P.;GEORGOPOULOS, V. C. An Fuzzy cognitive maps approach to process control systems. J. Advanced Computational Intelligence, n. 5, p. 1-9, 1999.

ZHANG, J. Y.; LIU, Z. Q.; ZHOU, S. Dynamic domination in Fuzzy causal network. IEEE Transactions on Fuzzy Systems, New York, v. 14, n. 1, p. 42-57, 2006. 
